\title{
Research of Air Pollution Dispersion Visualization Based on GPU and Volume Rendering
}

\author{
Lei Wang ${ }^{1, a}$ \\ ${ }^{1}$ College of Computer and Engineering, Wei Fang University , China \\ awanglpqpq@gmail.com
}

Keywords: Pollution Diffusion Model, GPU, Volume Rendering, Texture Mapping.

\begin{abstract}
To address the air pollution diffusing problem, a visualization algorithm based on fluid diffusion physical model and volume rendering is proposed to simulate the diffusion process epically for the urgent pollution accidents. The diffusion model is based on the simplified heat diffusion equation and the basic vortex constraint. The texture mapping based volume rendering method is adapted to handle the visualization algorithm. In addition, the render process adopts the GPU to implement the real-time visualization rendering.
\end{abstract}

\section{Introduction}

With the development of the modern industry and the accelerating of the urbanization process, the declining quality of the urban air becomes a global problem which attracts widespread attention. The primary causes of air pollution includes the surge increasing numbers of road-worthiness certificate, the continuous discharge of industrial pollution sources, the dense urban architecture and the crowded population. Therefore, the primary thing is to predict and control the air pollution. It will bring great harm once the sudden air pollution happens. In order to predict this class of sudden accidents in time and reduce the harm caused, the government should create constructing environmental monitoring and emergency treatment system positively. Pollution diffusion simulation and visualization is the core part of the environmental monitoring and emergency treatment system. Through the methods of visualization we can show the pollution pollutant concentration distribution. It is not only guiding persons to evacuate quickly, but also improve the efficiency of the emergency command.

Fluid simulation has developed rapidly in the application of computer graphics in the last ten years. The fluid simulation is used for lots of natural fluid phenomenon of visual simulation including smoke, water, cloud, foam, rivers. Stam[1] took the Semi-Lagrangian method into graphics to model smoke and cloud for the first time. Fedkiw[2] took the vortex constraint into graphics to model the details of smoke, which reveal the wonderful effect of smoke simulation. Foster [3] adopted the method of level set to create the surface of liquid in the water simulation. Harris[4] described the cloud simulation model which is based on physical in detail. In addition, the algorithm realizes real-time simulation rendering based on GPU. Feng Qiu[5] adopted the MRTLBM method to model flue gas diffusion and applied it to the safety application.

At present, there are not mature model to define the sudden air pollution diffusion simulation. Due to the affect of the environment on the sudden air pollution, it is need to simulate the whole process in time. In addition, the sudden air pollution diffuses in the local scope. So we need 3D visualization to simulate the vertical scale effect.

\section{Air Pollution Diffusion Model}

\section{Simplified diffusion model}

The process of air pollution diffusion is a complex procedure which is not only based on the basic fluid mechanics but also affected by rain, snow, sunshine and other weather conditions. In this paper, we focus on the sudden pollution accident simulation which includes the attributes of violent eruption, spread rapidly, area concentrated, easily affected and quickly dissipated. So the adapted diffusion model is proposed to address the above attributes. The attribute of the proposed algorithm 
is described as following:

1. Real-time Simulate. The simulated model should predict the diffusion result in time. Under the real-time simulated result, it is help to make the reasonable decision.

2. Simple model parameters. It is easy to access to the diffusion model parameters which include weather, terrain, buildings and pollutant.

3. Handle the complex building model. Because the pollution accidents often occur in built-up urban areas, the shape and distribution of the building has a great impact on the diffusion behavior. So the diffusion model should handle the complex building case.

Considering the attributes of sudden situation, the pollution diffusion model is defined according to the traditional flow simulation as following. The continuity equation is defined as the equation 1 . Due to the small viscosity of the air, we can ignore the viscosity item in the momentum equation. In this paper, the Momentum equation is defined as the following equation 2. In the above equations, the position variable parameters $\vec{u}, T, p$ respectively represent the speed, temperature and pressure. The constant parameters include the buoyancy coefficient $\alpha$ and $\beta$, ambient temperature $T_{a m b}$ and density $\rho$.

$$
\begin{gathered}
\nabla \cdot \vec{u}=0 \\
\frac{\partial \vec{u}}{\partial t}+\vec{u} \nabla \vec{u}=\left[\alpha s-\beta\left(T-T_{a m b}\right)\right] \vec{g}-\frac{1}{\rho} \nabla p
\end{gathered}
$$

The heat diffusion equation and concentration diffusion equation are defined as the following equation 3 and equation 4 . The related ambient data include the heat source parameter $S_{T}$ and pollution source parameter $S_{c}$.

$$
\begin{aligned}
& \frac{\partial T}{\partial t}+\vec{u} \cdot \nabla T=S_{T} \\
& \frac{\partial C}{\partial \mathrm{t}}+\vec{u} \cdot \nabla C=S_{c}
\end{aligned}
$$

\section{Turbulence modeling}

In the field of atmosphere, several of turbulence plays an implement impact on the spread of pollution air diffusion. The impact is over the role of molecular diffusion, and the air quality model existing now is basically designed for solving turbulence model. In 1994, [6] Steinhoff proposed vortex confined method (Vorticity Confinement) which is used for high Reynolds coefficient small scale turbulance modeling. So the vorticity confinement is used to define the turbulence modeling. Same with the Fedkiw 2001 method, the Momentum equation is defined as

the following equation 5 with the vorticity confinement constants. In the followed equation, $\vec{\omega}$ is defined as the curl of velocity field.

$$
\frac{\partial \vec{u}}{\partial t}+\vec{u} \nabla \vec{u}=\left[\alpha s-\beta\left(T-T_{a m b}\right)\right] \vec{g}+f_{v c}-\frac{1}{\rho} \nabla p
$$

\section{Experimental analysis}

According to the algorithm described in the paper, we firstly generate grid data about pollutant concentration of the related time by the physical simulation model mentioned above. Then, the visualization analysis and the show of relevant pollution data by the volume rendering method are based on GPU calculation. In the experiment, we adopt ordinary PC machine with the configuration: Core i5 3450 3.2G CPU, 4G memory, Nvidia GT640 graphics cards. 
In the experiment, $64 \times 64 \times 64$ grids are adopted with the internal of $3 \mathrm{~m}$. Meanwhile, wind speed is set $1.8 \mathrm{~m} / \mathrm{s}$ from left to right along the $\mathrm{X}$ axis. There are also several irregularly arranged rectangular obstacles. Pollutant concentration is in the range of from zero to one. The pollution source temperature is set 70 degrees. At the beginning, the speed is to $3 \mathrm{~m} / \mathrm{s}$, and concentration of the pollution source is 0.8 . In addition, there is a simulation on the related experimental grid data two minutes later.

Because of the large influence that the vortex constraint scale parameters have on pollutant diffusion behavior, three different vortex confined scales for visualization analysis experiment are adopted. In the process of volume rendering, the paper adopts different colors to describe pollutant concentration, with red representing high concentration and green indicating low concentration. In the whole experimental process, there are three different parameters adopted respectively for the effect of rending, including no vortex constraint, smaller scale vortex constraint (0.1) and larger scale vortex constraint (0.15). As shown in the figure 1 , there is the large scale vortex constraint (0.15).

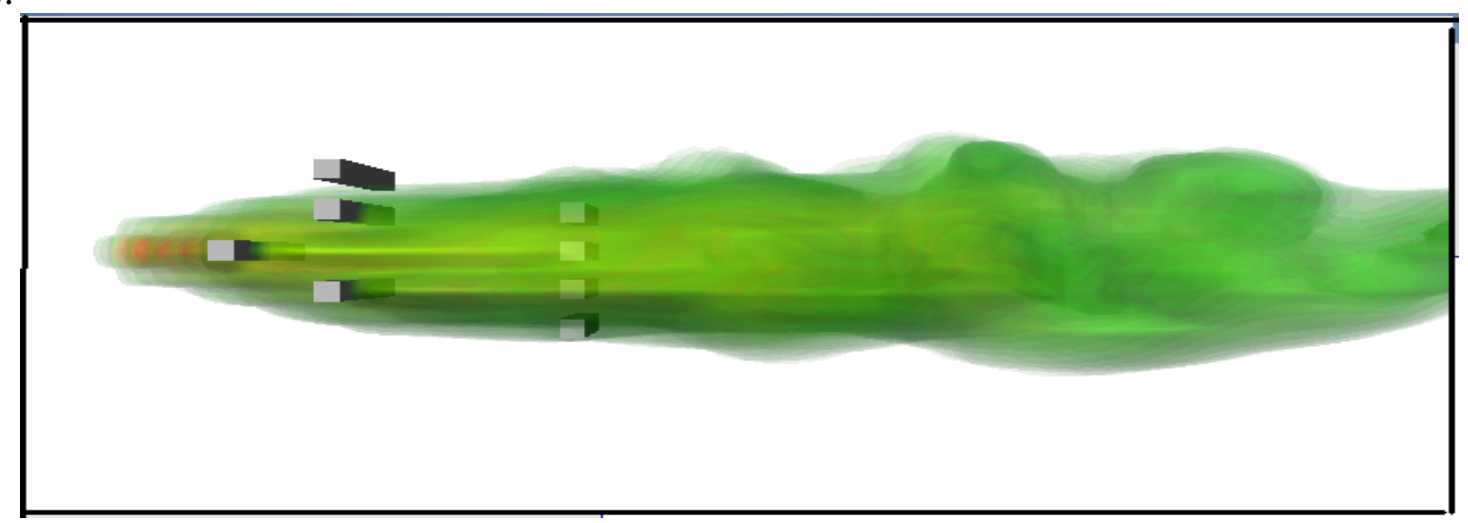

Fig. 1. Vertical view of larger scale vortex constraint with the illumination effect

\section{Conclusion}

According to the emergency simulation of the pollution accident, the paper has undergone an intensive study from three different respects: model design, visualization and interactive demonstration. The diffusion model is based on the simplified heat diffusion equation and the basic vortex constraint. The texture mapping based volume rendering method is adapted to handle the visualization algorithm. This method can generate real-time volume rendering effect using hardware texture mapping function.

\section{References}

[1] Jos Stam. Stable Fluids. ACM SIGGRAPH 1999.

[2] Ronald Fedkiw, Jos Stam. Visual Simulation of Smoke. ACM SIGGRAPH 2001

[3] Nick Foster and Ronald Fedkiw. Practical Animation of Liquids. ACM SIGGRAPH 2001

[4] Mark Jason Harris. Real-Time Cloud Simulation and Rendering. PhD dissertation. 2003

[5] Feng Qiu,Ye Zhao, Zhe Fan, Xiaoming Wei, Haik Lorenz, Jianning Wang,,

Suzanne Yoakum-Stover, Arie Kaufman, Klaus Mueller. Dispersion Simulation and Visualization for Urban Security. IEEE Visualization 2004

[6]J. Steinhoff and D. Underhill. Modification of the euler equations for "vorticity confinement”: Application to the computation of interacting vortex rings. Physics of

Fluids, 6(8):2738-2744, 1994 